\title{
Quis custodiet FAO?
}

\author{
In June 2019, the Food and Agriculture Organization of the United Nations will elect a new Director General, an \\ individual who will be central to global development for the next decade.
}

T he United Nations Sustainable Development Goals (SDGs) are an ambitious set of 17 targets, established in 2015 by the United Nations General Assembly, to inspire the improvement of the conditions of life on Earth by 2030, and possibly its very survival. They recognize that ending poverty and other deprivations must go hand-in-hand with strategies that improve health and education, reduce inequality, and spur economic growth - all while tackling climate change and working to preserve our oceans and forests. But setting goals is the easy part, somebody has to take responsibility for seeing them through.

The Food and Agriculture Organization (FAO) is one of the 'custodian organizations' charged with compiling and verifying country data and metadata, pertinent to the SDGs. The FAO is particularly responsible for SDG2, achieving 'zero hunger', but also components of five other SDGs relating to land, sea and water usage. And the picture is not good. The 2018 State of Food Security and Nutrition in the World (SOFI), produced by the FAO and other global agencies, reported the absolute numbers of hungry people rose for the third year in a row. These numbers have little to do with failure or success of plant-related and agricultural activities, and everything to do with increased conflict around the world, yet absolute numbers tell absolute stories of more hungry people.

Weighted by the burden of monitoring the SDGs' multiple indicators, the FAO is at the forefront of demonstrating if these goals are met, which can only happen through detailed and accurate reporting of agricultural and productivity statistics at a country level. This work is complex. Assessing global hunger is not as simple as politely knocking on doors and asking, 'are you hungry?'. It takes a much more nuanced approach to evaluate by proxy if a person receives enough calories to meet basic nutritional requirements, augmented with other metrics, such as nutrient quality and family income levels.

In 2017, while critiquing SDG2, the High Level Political Forum (HLPF) of the United Nations noted the lack of robust data and statistics for some of the indicators, stating that available evidence is limited, and urgent, scaled-up action is required to characterize small and medium producers. These data are needed to ensure the matching of local supply with local demand. The reports and datasets produced by the FAO statistics division (and made freely available at http://www.fao.org/faostat/) are relied on by analyses that shape global, national and local policies, which will be useless or worse if the raw statistics are not accurate.

Academic researchers are increasingly unconvinced that the FAO data is sufficient for its intended purposes. We are often told that FAO statistics are notoriously unreliable. If we do not trust the data, how can we trust the analysis? Work is underway to help improve the collection of statistics, but as noted above, such work is difficult, and even in our data-and-analytics hungry environment these are not the most headlinegrabbing of activities. Still, they need to be fully supported and funded for there to be any hope of the SDGs being attained.

Into the centre of this maelstrom of unattainable desires and underwhelming delivery will soon be stepping a new FAO Director General (DG), following what is arguably one of the most globally significant elections that will be held this year. They will be the ninth DG since the FAO was established at a conference in the dramatic Château Frontenac in Quebec, Canada, in 1945. Since the mid-1950s, FAO DGs have been elected to the role every four years but it has been normal for them to serve for two terms. Therefore, whoever succeeds the current incumbent, Braz José Graziano da Silva, can expect to be in office through to 2027, less than three years before the SDGs' deadline.

Candidates for the DG position are nominated by any one of the more than 190 member states and then elected on a onemember, one-vote basis in late-June of this year. By the closing date of 28 February 2019, there were five nominations. Firstly, there are two agricultural economists: Medi Moungui from Cameroon ${ }^{1}$, who is a former Chair of the Finance Committee of FAO; and, from India, Ramesh Chand who is currently a member of the National Institution for Transforming India. In addition, China has nominated their ViceMinister for Agriculture and Rural Affairs, Qu Dohgyu. Finally, there are two European candidates: AgroEngineer and former head of the European Food Safety Authority (EFSA), Catherine Geslain-Lanéelle from France; and Davit Kirvalidze, an agricultural scientist and former Georgian Minister for Agriculture.

That there is more than one nomination from Europe is a surprise. In 2011, there was both a Spanish and an Austrian nominee; the former of which, Miguel Ángel Moratinos, narrowly lost out to da Silva, 88 votes to 92 , in the second round of voting. It was thought that competition between these two nominees had hampered them both and so reduced the chances of a European heading the organisation. Then at the 2015 elections, European states failed to nominate anyone by the deadline, allegedly due to failures in communication. This year, members of the European Union (EU) agreed to an informal process and selected Geslain-Lanéelle as an EU candidate. However, Georgia, while it has an Association Agreement with the EU and some desire to join, is not a member.

Whoever takes over as DG of FAO later this year will be faced with vast and myriad challenges, not merely the collection of crucial statistics in agriculture. The FAO's website lists over 90 specific projects ranging from the very specific, such as the prevention and control of Bovine Paralytic Rabies, to those with much broader focus, such as e-Agriculture strategies or initiatives to show the quality of food products through traceability of their origins.

The calibre of candidates for the FAO top job is clear. What is less certain is in what directions they might take the organization. Given the method of election for this post, there is little reason for the candidates to make public statements. Nevertheless, Chatham House, the Royal Institute of International Affairs, has organized an event on 12 April 2019 in Rome with at least two of the nominees, to "encourage the candidates to set out their vision for the FAO in front of a wider public audience" (https://go.nature.com/2Ufml0A).

The FAO was established to raise standards of nutrition and living, and in a forechoing of SDG2, ensure 'humanity's freedom from hunger.' The incoming DG, whoever that may be, would do well to remember that Article 1 of its founding constitution exhorts it to "collect, analyse, interpret and disseminate information relating to nutrition, food and agriculture" (https://bit. ly/2WrgFOC). Nothing has changed. Good policy-making will always rely on accurate, transparent and appropriate information.

${ }^{1}$ Mr Médi Moungui announced the withdrawal of his candidature in a 21 March 2019 letter addressed to the Secretary-General of the Conference and the Council of FAO.]

Published online: 8 April 2019 https://doi.org/10.1038/s41477-019-0417-9 